Journal of Computer Science 4 (11): 910-915, 2008

ISSN 1549-3636

(C) 2008 Science Publications

\title{
A Robust Video Watermarking Algorithm
}

\author{
${ }^{1}$ Tahani Al-Khatib, ${ }^{2}$ Ali Al-Haj, ${ }^{1}$ Lama Rajab and ${ }^{1}$ Hiba Mohammed \\ ${ }^{1}$ The University of Jordan, Al-Jubeiha, 11942 Amman, Jordan \\ ${ }^{2}$ Princess Sumaya University for Technology, P.O. Box 1928, Al-Jubeiha, 11941, Jordan
}

\begin{abstract}
Problem statement: Video watermarking is well known as the process of embedding copyright information in video bit streams. It had been proposed in recent years to solve the problem of illegal manipulation and distribution of digital video. Approach: In this study, an effective, robust and imperceptible video watermarking algorithm was proposed. This algorithm was based on a cascade of two powerful mathematical transforms; Discrete Wavelets Transform (DWT) and Singular Value Decomposition (SVD). Two different transform domain techniques showed high level of complementary and different levels of robustness against the same attack will be achieved through their combination. Results: The proposed algorithm was tested against imperceptibility and robustness and excellent results were obtained. Conclusion: Experimental results demonstrate the robustness achieved by combining the two transforms.
\end{abstract}

Key words: Digital video watermarking, copyright protection, Discrete Wavelets Transform (DWT), Singular Value Decomposition (SVD), imperceptible and robust watermarking

\section{INTRODUCTION}

Digital video is becoming popular more than ever due to the widespread of video-based applications such as Internet video, videophones, wireless video, video conferencing, among many others. However, a byproduct of such popularity is the worldwide unauthorized copying and distribution of digital video. Digital watermarking has been proposed in recent years to prevent illegal and malicious copying and distribution of digital media by embedding an unnoticeable information (called a watermark) into the media content ${ }^{[1]}$.

The watermark is usually a copyright message identifying the ownership information of the media object. Effective watermarking has many requirements, the most important of which are imperceptibility and robustness. Imperceptibility refers to perceptual transparency and it requires that the watermarking algorithm to embed the watermark in such a way that the quality of the underlying video frames is not affected. As for the robustness requirement, the watermark must always remain in the watermarked video frames, even if the quality of the frames is degraded intentionally or unintentionally ${ }^{[2]}$.

Current video watermarking techniques can be grouped into two major classes; spatial-domain watermarking techniques and watermarking frequencydomain techniques. Spatial-domain techniques embed a watermark in the frames of a given video by modifying its pixels directly. These techniques are easy to implement and require few

Computational resources, however, they are not robust against common digital signal processing operations such as video compression ${ }^{[3]}$. On the other hand, transform-domain watermarking techniques modify the coefficients of the transformed video frames according to a pre-determined embedding scheme. The scheme disperses the watermark in the spatial domain of the video frame ${ }^{[4]}$, hence making it very difficult to remove the embedded watermark. Compared to spatialdomain techniques, frequency-domain watermarking techniques proved to be more effective with respect to achieving the imperceptibility and robustness requirements of digital watermarking algorithms.

In this study, we propose a blind, imperceptible and robust video watermarking technique. The algorithm is based on cascading two powerful mathematical transforms; the Discrete Wavelet Transform (DWT) ${ }^{[5]}$ and the Singular Value Decomposition $(\mathrm{SVD})^{[6]}$. The two transforms are different transform domain techniques and thus provide different, but complementary, levels of robustness against the same attack. More robustness is expected by combining benefits of the two transforms. In the proposed hybrid algorithm, the watermark bits are not embedded directly on the wavelet coefficients, but rather on the elements of singular values of the frames' DWT sub-bands. 
The proposed algorithm is also 'blind' in the sense that it does not require the original video in order to extract the embedded watermark.

The objectives of this study were to: (i) achieve more robustness and imperceptibility against attacks by combining benefits of SVD and DWT methods, (ii) achieve flexibility and effectiveness in extraction the watermark from video frames with no need of the original video and (iii) Compare the proposed algorithm performance of four different videos scenes, with different watermarks and for various video attacks.

\section{MATERIALS AND METHODS}

\section{Mathematical materials:}

The discrete wavelets transform: Wavelets are special functions which, in a form analogous to sines and cosines in Fourier analysis, are used as basal functions for representing signals. For 2-D images, applying DWT corresponds to processing the image by 2-D filters in each dimension. The filters divide the input image into four non-overlapping multi-resolution subbands LL, LH, HL and HH. The LL sub-band represents the coarse-scale DWT coefficients while the LH, HL and HH sub-bands represent the fine-scale DWT coefficients. To obtain the next coarser scale of wavelet coefficients, the LL sub-band is further processed until some final scale $\mathrm{N}$ is reached. When $\mathrm{N}$ is reached, we will have $3 \mathrm{~N}+1$ sub-bands consisting of the multi-resolution sub-bands $\mathrm{LL}_{\mathrm{N}}$ and $\mathrm{LH}_{\mathrm{x}}, \mathrm{HL}_{\mathrm{x}}$ and $\mathrm{HH}_{\mathrm{x}}$ where $\mathrm{x}$ ranges from 1 until N. Due to its excellent spatio-frequency localization properties, the DWT is very suitable to identify areas in the host image where a watermark can be embedded effectively ${ }^{[7-9]}$. In particular, this property allows the exploitation of the masking effect of the human visual system such that if a DWT coefficient is modified, only the region corresponding to that coefficient will be modified.

Singular value decomposition: The DWT transform attempts to decompose an image in terms of a standard basis set. This needs not necessarily be the optimal representation for a given image. On the other hand, Singular Value Decomposition (SVD) is a numerical technique for diagonalizing matrices in which the transformed domain consists of basis states that is optimal in some sense. The SVD of an $\mathrm{N} x \mathrm{~N}$ matrix A is defined by the operation:

$$
\mathrm{A}=\mathrm{US} \mathrm{V}^{\mathrm{T}}
$$

where, $\mathrm{U}$ and $\mathrm{V} \in \mathrm{R}^{\mathrm{N} \times \mathrm{N}}$ are unitary and $\mathrm{S} \in \mathrm{R}^{\mathrm{N} \times \mathrm{N}}$ is a diagonal matrix. The diagonal entries of $S$ are called the singular values of $\mathrm{A}$ and are assumed to be arranged in decreasing order $\sigma_{i}>\sigma_{i}+1$. The columns of the $U$ matrix are called the left singular vectors while the columns of the $\mathrm{V}$ matrix are called the right singular vectors of $A$. Each singular value $\sigma_{i}$ specifies the luminance of an image layer while the corresponding pair of singular vectors specifies the geometry of the image layer ${ }^{[10-12]}$. In SVD-based watermarking, an image is treated as a matrix decomposed by SVD into the three matrices; U, S and $\mathrm{V}^{\mathrm{T}}$, as shown in Fig. 1. By virtue of the fact that slight variations in the elements of matrix $S$ does not affect visual perception of the quality of the cover image, most existing SVD-based watermarking algorithms add the watermark information to the singular values of the diagonal matrix $\mathrm{S}$ in such a way to meet the imperceptibility and robustness requirements of effective digital image watermarking algorithms.

\section{Proposed method:}

DWT-SVD video watermarking algorithm: The proposed DWT-SVD watermarking algorithms consist of two procedures, the first embeds the watermark into the original video clip, while the other extracts it form the watermarked version of the clip.

Watermark embedding procedure: The embedding procedure is depicted in the block diagram shown in Fig. 2.

Step 1: Divide the video clip into video scenes $\mathrm{Vs}_{\mathrm{i}}$.

Step 2: Process the frames of each video scene using DWT and SVD as described in steps 3-10.

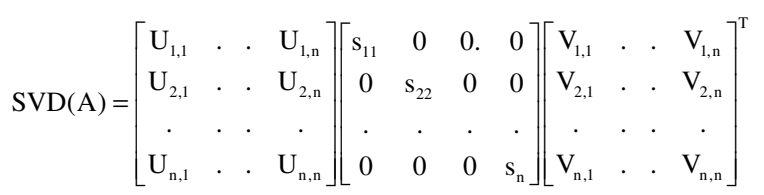

Fig. 1: The SVD operation SVD $(A)=U^{S ~} V^{T}$

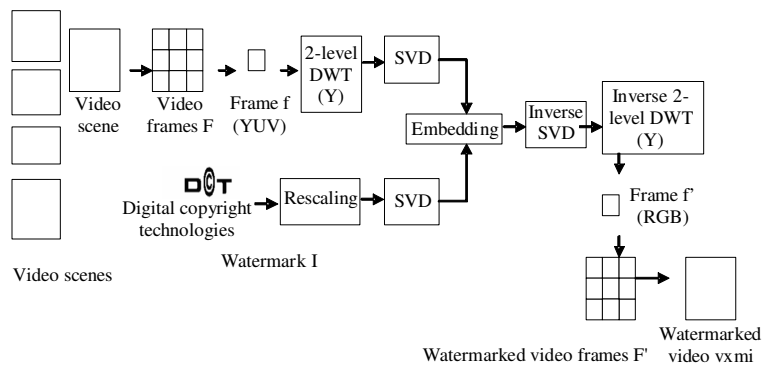

Fig. 2: DWT-SVD watermark embedding procedure 


\begin{tabular}{|c|c|c|}
\hline \multirow{2}{*}{$\mathrm{LL}_{1}$} & $\mathrm{LL}_{2}$ & $\overline{\mathrm{FL}_{2}}$ \\
\cline { 2 - 3 } & $\mathrm{LH}_{2}$ & $\mathrm{HH}_{2}$ \\
\hline & & \\
$\mathrm{LH}_{1}$ & \multicolumn{2}{|c|}{$\mathrm{HH}_{1}$} \\
\hline
\end{tabular}

Fig. 3: Frame's 2-level DWT sub-bands

Step 3: Convert every video frame $F$ from RGB to YUV color matrix format.

Step 4: Compute the 2-level DWT for the Y (luminance) matrix in each frame $\mathrm{F}$. This operation generates seven DWT sub-bands $\left[\mathrm{LL}_{1}, \mathrm{LL}_{2}, \mathrm{HL}_{2}, \mathrm{LH}_{2}\right.$, $\left.\mathrm{HH} 2, \mathrm{LH}_{1}, \mathrm{HH}_{1}\right]$. Each sub-band is a matrix of DWT coefficients at a specific resolution. Figure 3 shows the sub-bands produced by the 2-level DWT decomposition.

Step 5: Apply the SVD operator on the $\mathrm{HL}_{2}$ sub-band. The SVD operator decomposes the sub-band's coefficient matrix into three independent matrices:

$\mathrm{HL}_{2}=\mathrm{U}_{\mathrm{HL} 2} \mathrm{~S}_{\mathrm{HL} 2} \mathrm{~V}_{\mathrm{HL} 2}$.

Step 6: Rescale the watermark image so that the size of the watermark will match the size of the $\mathrm{HL}_{2}$ sub-band which will be used for embedding.

Step 7: Embed the binary bits of watermark $\mathrm{W}_{\mathrm{Vsi}}$ into $\mathrm{S}_{\mathrm{HL} 2}$ by substituting the watermark bit $\mathrm{W}_{\mathrm{i}}$ with the LSB (Least significant Bit) bit of $\mathrm{S}_{\mathrm{HL} 2}(\mathrm{i}, \mathrm{i})$ :

$\operatorname{LSB}\left(\mathrm{S}_{\mathrm{HL} 2}(\mathrm{i}, \mathrm{i})\right)=\mathrm{W}_{\mathrm{Vsi}}$

Step 8: Apply the inverse SVD operator on the modified $S_{\mathrm{HL} 2}$ matrix to get a modified coefficient matrix HL2'. The inverse SVD operation is as follows:

$\mathrm{HL} 2^{\prime}=\mathrm{U}_{\mathrm{HL} 2} \mathrm{~S}_{\mathrm{HL} 2} \mathrm{~V}_{\mathrm{HL} 2}{ }^{\mathrm{T}}$

Step 9: Apply the inverse DWT on the modified coefficient matrix HL2. This operation produces the final watermarked Video frame F.

Step 10: Convert the video frames F' from YUV to RGB color matrix.

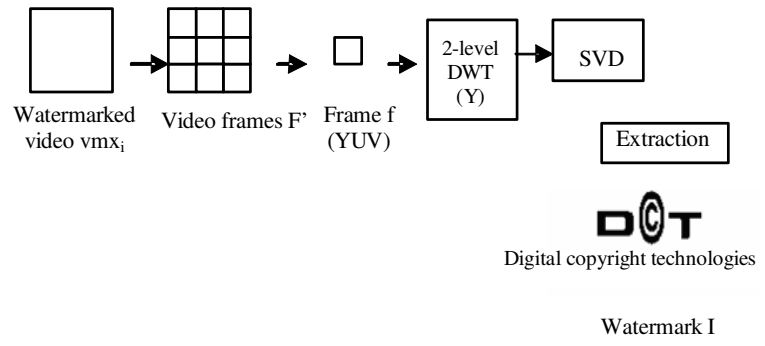

Fig. 4: DWT-SVD watermark extraction procedure

Step 11: Reconstruct frames into the final watermarked Video scene $\mathrm{Vs}_{\mathrm{i}}$ '.

Step 12: Reconstruct watermarked scenes to get the final watermarked Video clip.

Watermark extraction procedure: The proposed DWT-SVD algorithm is blind in the sense that it does not need the original video in the extraction process. Therefore, we can extract the watermark image from the watermarked video frames from the LSBs directly as depicted in the block diagram shown in Fig. 4 and described in details in the steps that follow:

Step 1: Divide the watermarked Video clip V' into watermarked scenes Vsi'.

Step 2: Process the watermarked frames of each watermarked video scene using DWT and SVD as described in steps $3 \sim 6$.

Step 3: Convert the video frame $F^{\prime}$ from RGB color matrix to YUV.

Step 4: Compute the 2-level DWT for the frame F. Let the seven sub-bands produced after this process be:

$\left[\mathrm{wLL}_{1}, \mathrm{wLL}_{2}, \mathrm{wHL}_{2}, \mathrm{wLH}_{2}, \mathrm{wHH}_{2}, \mathrm{wLH}_{1}, \mathrm{wHH}_{1}\right]$

Step 5: Apply the SVD operator on the $\mathrm{wHL}_{2}$ subband. The SVD operator decomposes the sub-band's coefficient matrix into three independent matrices:

$\mathrm{wHL}_{2}=\mathrm{U}_{\mathrm{wHL} 2} \mathrm{~S}_{\mathrm{wHL} 2} \mathrm{~V}_{\mathrm{wHL} 2}$.

Step 6: Extract the embedded watermark from the diagonal matrix $S_{\mathrm{wHL} 2}$ as follows:

$\mathrm{W}_{\mathrm{Vsi}}(\mathrm{i})=\mathrm{LSB}\left(\mathrm{S}_{\mathrm{HL} 2}(\mathrm{i}, \mathrm{i})\right)$

Step 7: Construct the image watermark $\mathrm{W}_{\mathrm{Vsi}}$ by cascading all watermark bits extracted from all frames. 
Step 8: Repeat the same procedure for all video scenes.

\section{RESULTS}

We evaluated the performance of our proposed DWT-SVD video watermarking algorithm using a colored video clip having a size of 351 frames. Each frame has a size of $240 \times 352$ pixels. The video clip was partitioned into four scenes having the size of 101,74,102 and 75, frames, respectively. A snap shot of each scene is shown in Fig. 5. The watermark was then embedded in the Y component each frame of all scenes.

A different watermark was embedded in each of the four scenes. The four watermark images are shown in Fig. 6. Each watermark consists of $72 \times 181$ pixels.
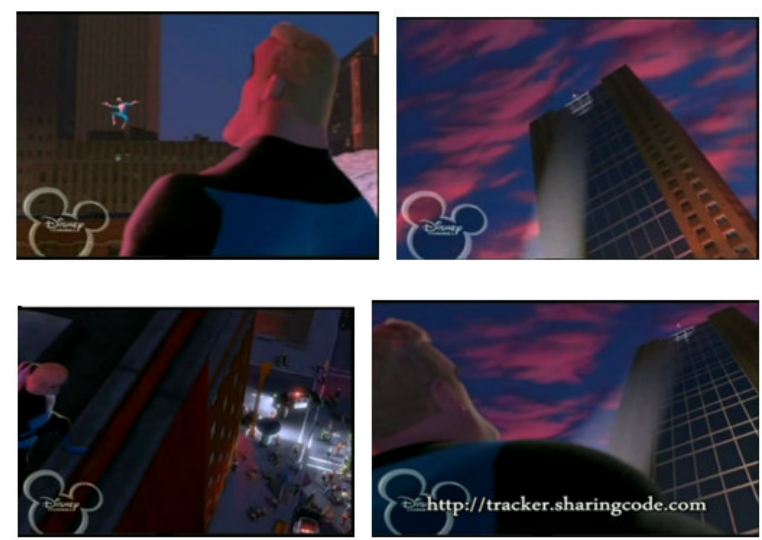

Fig. 5: Snapshots of the four scenes in the video clip

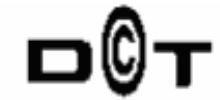

Digital copyright technologies
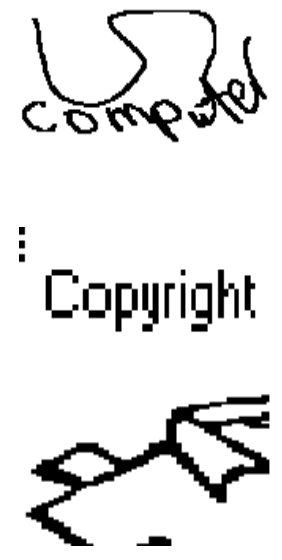

Fig. 6: The four different watermarks embedded in the scenes of the video clip
We evaluated the performance of the proposed DWT-SVD video watermarking algorithm with respect to imperceptibility and robustness ${ }^{[12]}$. Results are presented and discussed in Fig. 7-11.

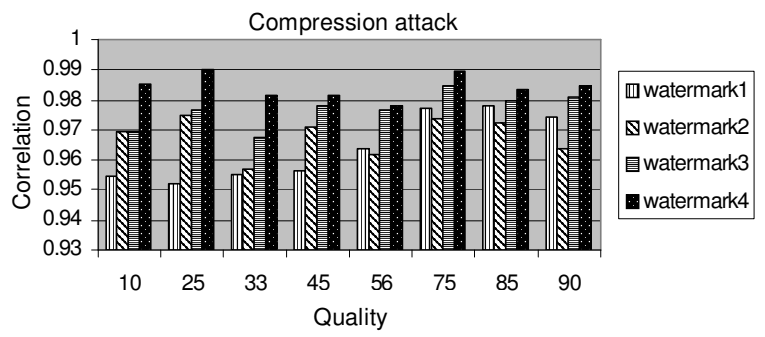

Fig. 7: Correlation values due to different rates of frame compression

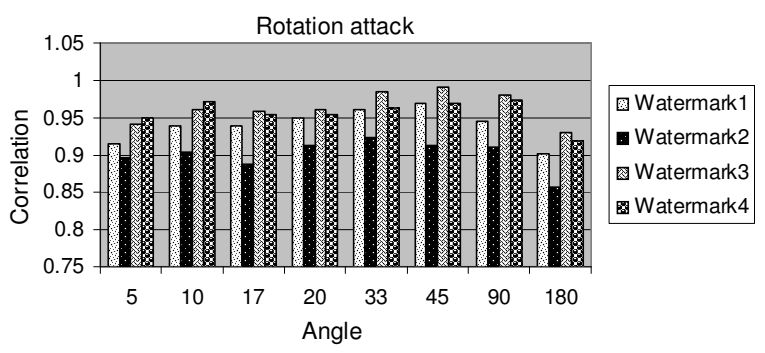

Fig. 8: Correlation values due to frame rotations at different angles

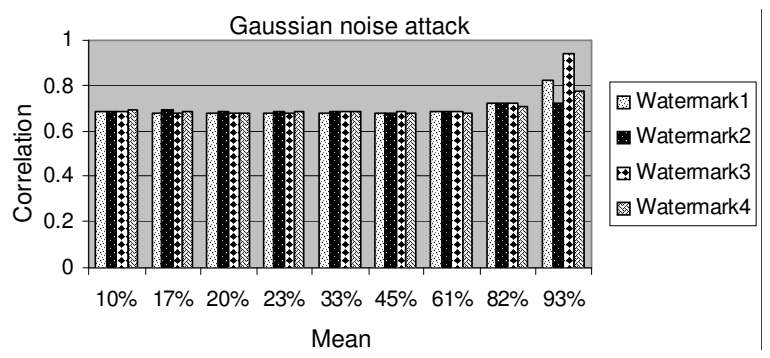

Fig. 9: Correlation values due to the of Gaussian noise

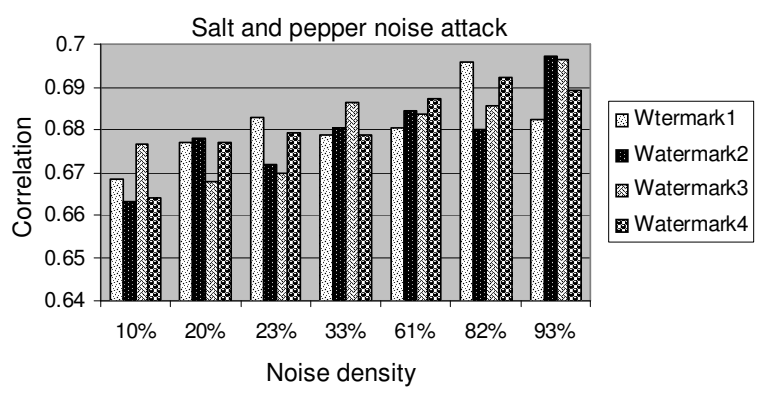

Fig. 10: Correlation values due to the addition of Salt and pepper noise 


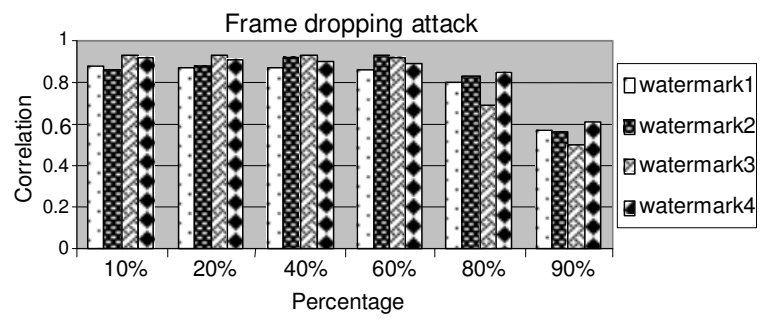

Fig. 11: Correlation values due to video frame dropping

\section{DISCUSSION}

Imperceptibility: Imperceptibility means that the perceived quality of the video clip should not be distorted by the presence of the watermark. As a measure of the quality of a watermarked video, the Peak Signal to Noise Ratio (PSNR) is typically used. In our study, the watermark was embedded in the video according the procedure described. The average PSNR for the all frames of the four watermarked scenes was 48.1308. This high PSNR value proves imperceptibility of the proposed SVD-DWT algorithm.

Robustness: Robustness is a measure of the immunity of the watermark against attempts to remove it or degrade it by different types of digital signal processing attacks. We measured the similarity between the original watermark and the watermark extracted from the attacked watermarked images using the correlation factor $\rho$ which is computed using Eq. 7 given:

$$
\rho(w, \hat{w})=\frac{\sum_{i=1}^{N} w_{i} \hat{w}_{i}}{\sqrt{\sum_{i=1}^{N} w_{i}^{2}} \sqrt{\sum_{i=1}^{N} \hat{w}_{i}^{2}}}
$$

Where:

$\mathrm{N}=$ The number of pixels in watermark

$\mathrm{w}$ and $\hat{\mathrm{w}}=$ The original and extracted watermarks respectively. The correlation factor $\rho$ may take values between $0-1$

In Fig. 5-8 we show the robustness results obtained for four standard attacks: rotation, JPEG compression, Gaussian noise and salt and pepper noise ${ }^{[13]}$. The high correlation values obtained for all attacks clearly indicate the robustness of the algorithm against standard attacks. Results and a brief description of each attack.

Video compression: The watermarked video frames were compressed with different quality factors. As shown in Fig. 7, the correlation values indicate clearly robustness of the algorithm against the video compression.

Video rotation: The watermarked video frames were rotated with different angles. As shown in Fig. 8, the correlation values indicate clearly robustness of the algorithm against the video frames rotation.

Gaussian noise: A Gaussian noise was added with varying intensities to the watermarked video frames. As shown in Fig. 9, the correlation values indicate clearly robustness of the algorithm against the addition of Gaussian noise.

Salt and pepper noise: A salt and pepper noise was added with varying intensities to the watermarked video frames. As shown in Fig. 10, the correlation values indicate clearly robustness of the algorithm against the addition of salt and pepper noise

Other than the standard attacks, we evaluated robustness due to video frame dropping ${ }^{[14]}$. The attackers hope by performing such an attack that the embedded watermark will be degraded or removed without hindering the original video. This is due to the fact that large amount of redundancy. Exist between video frames and therefore video dropping should leave the integrity of the original video intact. The results are shown in Fig. 11. As seen, even if the attacker drops $60 \%$ of the frames, the watermark can still be extracted with a correlation value.

\section{CONCLUSION}

In this study, we proposed a novel digital video watermarking algorithm. The algorithm makes use of two powerful mathematical transforms; DWT and SVD. Both techniques were combined to exploit their attractive features; spatio-frequency localization of the DWT and compact capturing of semi-global features and the geometric information of images by the significant components of the SVD. Experimental results demonstrated the blindness and robustness of our proposed method as it successfully extracted the watermark from each frame without using the original video. The extracted watermark was exactly the same as the embedded original watermark.

\section{ACKNOWLEDGMENT}

The researchers acknowledge the financial support received from the CIS department in the University of Jordan and PSUT University. 


\section{REFERENCES}

1. Langelaar, G., I. Setyawan and R. Lagendijk, 2000. Watermarking digital image and video data: A state-of-art overview. IEEE Signal Process. Mag., 17: 20-46.

http://ieeexplore.ieee.org/xpls/abs_all.jsp?arnumbe $\mathrm{r}=879337$

2. Doerr, G. and J. Dugelay, 2003. A guided tour to video watermarking. Signal Process. Image Commun., 18: 263-282.

http://eprints.adastral.ucl.ac.uk/5/2/imageComm2003.pdf

3. Hartung, H. and B. Girod, 1998. Watermarking of compressed and un-compressed video. Signal Process., 66: 283-301. DOI: 10.1016/S01651684(98)00011-5

4. Chan, P. and M. Lyu, 2003. A DWT-based digital video watermarking scheme with error correcting code. Lecture Notes Comput. Sci., 2836: 202-213. DOI: $10.1007 / \mathrm{b} 13930$

5. Mallat, S., 1989. A theory for multi-resolution signal decomposition: The wavelet representation. IEEE Trans. Patt. Anal. Mach. Intel., 11: 674-693. http://www.cmap.polytechnique.fr/ mallat/papiers/ MallatTheory89.pdf

6. Andrews, H. and C. Patterson, 1976. Singular value decompositions and digital image processing. IEEE Trans. Acoust. Speech Signal Process., 24: 26-53. $\mathrm{http} /$ /ieeexplore.ieee.org/xpl/freeabs_all.jsp?arnum ber $=1162766$

7. Gao, X. and X. Tang, 2002. Unsupervised videoshot segmentation and model-free anchorperson detection for news video story parsing. IEEE Trans. Circ. Syst. Video Technol., 12: 765-776. http://ieeexplore.ieee.org/stamp/stamp.jsp?arnumbe $\mathrm{r}=01031915$
8. Reddy, A. and B. Chatterji, 2005. A new wavelet based logo-watermarking scheme. Patt. Recog. Lett., 26: 1019-1027. DOI: 10.1016/J.PATREC.2004.09.047

9. Liu, R. and T. Tan, 2002. A SVD-based watermarking scheme for protecting rightful ownership. IEEE Trans. Multimedia, 4: 121-128. DOI: 10.1109/ TMM.2006.886297

10. Chang, C., P. Tsai and C. Lin, 2005. SVD-based digital image watermarking scheme. Patt. Recog. Lett., 26 : 1577-1586. DOI: 10.1016/j.patrec.2005.01.004

11. Wu, Y., 2005. On the security of SVD-based ownership watermarking. IEEE Trans. Multimedia, 7: 624-627. DOI: 10.1109/TMM.2005.846774

12. Petitcolas, F.A.P. and R.J. Anderson, 1999. Evaluation of copyright marking systems. Proceeding of the IEEE International Conference on Multimedia Computing and Systems, June 7-11, Florence, Italy, pp: 1-6. http://www.petitcolas.net/fabien/publications/ieee mm99-evaluation.pdf

13. Voloshynovskiy, S., S. Pereira and T. Pun, 2001. Attacks on digital watermarks: Classification, estimation-based attacks and benchmarks. Commun. Mag., 39: 118-126. DOI: 10.1.1.84.5061

14. Kundur, D., K. Su and D. Hatzinakos, 2004. Digital Video Watermarking: Techniques, Technology and Trends. In: Intelligent Watermarking Techniques, Pan, P.J.S., H.C. Huang and L. Jain (Eds.). World Scientific Publishing Company, ISBN: 9812387579, pp: 852. 